\title{
Facilitation by repetition in recognition memory for tonal pitch
}

\author{
DIANA DEUTSCH \\ Center for Human Information Processing, University of California at San Diego, La Jolla, California 92037
}

\begin{abstract}
Subjects made delayed pitch comparisons when the standard and comparison tones were separated by a sequence of interpolated tones. In some conditions, a tone of the same pitch as the standard tone was included among the interpolated tones. Recognition performance was superior for sequences where the standard tone pitch was repeated, even compared with control sequences of reduced size. The improvement in performance produced by the repeated tone depended on its position in the intervening sequence. Improvement was substantial and highly significant when the standard tone pitch was repeated in the second serial position of a sequence of six interpolated tones, but small and insignificant when it was repeated in the fifth serial position.
\end{abstract}

It is generally the case in verbal memory that repeating an item in a list results in superior retention for that item. The probability of free recall for a verbal item increases as a monotonic function of the number of its occurrences in a list (Hintzman, 1970; Underwood, 1969; Waugh, 1962). A similar effect is found for recognition performance (Hintzman, 1970). When ordered recall is required, performance on repeated items may show a decrement (Crowder \& Melton, 1965; Wolf \& Jahnke, 1968); however, superior recognition of these repeated items nevertheless occurs using the same stimuius materials (Wolf \& Jahnke, 1968).

In contrast, very little is known about the effect of repetition on memory for nonverbal materials. Several experimenters have used the Sperling (1960) partial report paradigm to determine whether iconic memory is susceptible to enhancement by repetition. These studies have produced conflicting results. Turvey (1967) obtained partial reports on arrays of digits exposed tachistoscopically for durations of $50 \mathrm{msec}$. A given slide was repeatedly presented in alternation with nonrepeated slides, and no effect of repetition was found. However, Besner, Keating, Cake, and Maddigan (1974) used a similar paradigm, but with smaller stimulus arrays of $100-\mathrm{msec}$ duration, and did obtain superior partial report of repeated slides compared with nonrepeated slides. Further, Standing and Da Polito (1968) studied the effect of repeating a single row, in a randomly varying position, in several successive arrays. No repetition effect was found when report from another row of the array was required on repetition trials; however, performance did improve with repetition when the subjects were asked to attend without response either to another row or to the whole array on repetition

This work was supported by United States Public Health Service Grant MH-21001-03. I thank J. Russo for statistical discussions.

Requests for reprints should be sent to Diana Deutsch, Department of Psychology, University of California at San Diego, P. O. Box 109, La Jolla, California 92037. trials. It appears from these various studies that repetition effects in iconic memory are very sensitive to the experimental variables employed.

The partial report procedure studies memory for information which, due to lack of time and to the limitations imposed by the selective attention mechanism, has not been translated into verbal form. In contrast, one may inquire into the effects of repetition in nonverbal memory where each item is fully perceived and attended to, but where, due to the nature of the stimulus materials, verbal encoding does not take place. In a previous study, Deutsch (1972) obtained evidence for facilitation by repetition in such memory. A standard tone was presented, followed by a sequence of interpolated tones, and then by a comparison tone. The subjects were required to judge whether the comparison tone was the same or different in pitch compared with the standard tone. Repeating the standard tone pitch among the interpolated tones resulted in a substantial improvement in performance, both when the standard and comparison tones were the same in pitch and when they differed.

One might, however, argue that this apparent memory enhancement due to repetition was based on an artifact. The subjects may have recognized the repetition of the standard tone in the sequence, and so adopted the repeated tone as the new standard. In this way, the effective interval between the standard tone and the comparison tone would have been reduced, as would the effective number of interpolated tones. This could then explain the superior performance in such sequences. The first experiment was designed to rule out this possibility. Performance was compared for sequences containing repeated tones, for sequences of the same size containing no repeated tones, and for sequences containing no repeated tones which were reduced in size, so that a retention interval of constant duration and a constant number of interpolated tones separated the most recent presentation of the standard tone from the comparison 
Table 1

Mean Percentages of Errors in Conditions of Experiment I

\begin{tabular}{|c|c|c|}
\hline & \multicolumn{2}{|c|}{ Percent Errors } \\
\hline & \multicolumn{2}{|c|}{$\begin{array}{c}\text { Standard and } \\
\text { Comparison Tones }\end{array}$} \\
\hline Condition & Same & Different \\
\hline $\begin{array}{l}\text { A. Six interpolated tones; stan- } \\
\text { dard tone pitch repeated in } \\
\text { second serial position. }\end{array}$ & .32 & 1.60 \\
\hline $\begin{array}{l}\text { B. Six interpolated tones; stan- } \\
\text { dard tone pitch not repeated. }\end{array}$ & 12.50 & 13.14 \\
\hline $\begin{array}{l}\text { C. Four interpolated tones; } \\
\text { standard tone pitch not } \\
\text { repeated. }\end{array}$ & 6.09 & 10.90 \\
\hline
\end{tabular}

tone. If true memory facilitation were produced by repeating the standard tone pitch, recognition performance should be superior for sequences containing repeated tones than for the other sequences, even of reduced size.

\section{EXPERIMENT I}

\section{Method}

Procedure. The following procedure was used. The subjects listened to a standard tone, which was followed by a set of intervening tones, and then, after a pause, by a comparison tone. The subjects were told to remember the standard tone, to ignore the intervening tones, and then to judge whether the comparison tone was the same or different in pitch compared to the standard tone. The subjects recorded their judgments by writing ' $S$ ' (same) or ' $D$ ' (different) on paper.

Temporal Parameters. All tones were $200 \mathrm{msec}$ in duration. The interval between the standard tone and the first intervening tone was $300 \mathrm{msec}$, and the intervening tones were also spaced 300 msec apart. A 2-sec pause was incorporated between the last intervening tone and the comparison tone. Sequences were presented in groups of 12 . Sequences within each group were separated by $10-\mathrm{sec}$ pauses; a 5 -min pause was incorporated between groups.

Conditions. The experiment consisted of three conditions. In the first two conditions, the standard and comparison tones were separated by a sequence of six intervening tones. In Condition $A$, a tone which was identical in pitch to the standard tone was included in the second serial position of the intervening sequence. In Condition $B$, no such tone was included. In Condition $\mathrm{C}$, the standard and comparison tones were separated by a sequence of four intervening tones; no tone which was identical in pitch to the standard tone was included in the intervening sequence. Thus in Conditions $\mathrm{A}$ and $\mathrm{C}$ a retention interval of identical duration and an identical number of intervening tones separated the comparison tone from the most recent presentation of the standard tone pitch.

Each condition consisted of 24 sequences. In half of these sequences the standard and comparison tones were the same in pitch, and in the other half they differed. Within each condition, when the standard and comparison tones differed, in half of the instances the standard tone was higher than the comparison tone, and in the other half it was lower. The sequences were presented in random order, with no separation by condition, except that no two adjacent sequences contained the same standard or comparison tone pitches, so as to minimize repetition effects across sequences. The subjects listened to the entire tape on two separate days, and the results for each individual subject were averaged.
Tonal Stimuli. The standard and comparison tones were taken from an equal-tempered scale (International Pitch; $A=435$;) and ranged from the $\mathrm{C \#}$ above middle $\mathrm{C}$ to the $\mathrm{C}$ an octave above. The following frequencies were therefore employed (in $\mathrm{Hz}$ ): $\mathrm{C} \#=274, \mathrm{D}=290 . \mathrm{D} \#=308, \mathrm{E}=326, \mathrm{~F}=345, \mathrm{~F} \#=366$, $\mathrm{G}=388, \mathrm{G} \#=411, \mathrm{~A}=435, \mathrm{~A} \#=461, \mathrm{~B}=488$ and $\mathrm{C}=517$. In all conditions each of these pitches was used equally often, either as a standard tone, or as a comparison tone, or both.

The intervening tones were chosen randomly from the set specified above with two restrictions. First, no intervening sequence contained a tone of the same pitch as the standard or comparison tone of that sequence, except as specified by the experimental contitions. Second, no sequence contained more than one example of any tonal pitch.

Subjects. Thirteen paid undergraduates at the University of California at San Diego served as subjects in this experiment. These were selected on the basis of obtaining a score of at least $80 \%$ correct on a short tape containing sequences designed as in Condition B (i.e., in which a sequence on six tones was interpolated between the standard and comparison tones, and the standard tone pitch was not repeated).

Apparatus. The tones were generated as sine waves by a Wavetek oscillator controlled by a PDP/8 computer, and the output was recorded on high fidelity tape. The tape was played to subjects on a high quality tape recorder, the output of which was passed through a frequency balance control (Advent Corp.) and a Fisher stereo master control amplifier, with the controls adjusted so that the tones were equal in apparent loudness. The output was played to subjects through high quality loudspeakers.

\section{Results and Discussion}

The percentage errors in each experimental condition were computed separately for each subject, averaged over the two experimental sessions, both for sequences in which the standard and comparison tones were the same in pitch and for sequences where they differed. Table 1 shows the percentage errors for each of these conditions, averaged over the whole group of subjects. It can be seen that the error rates for sequences where the standard tone was repeated were substantially lower than for sequences containing no repeated tones. This was true both when the sequences containing no repeated tones were of the same size and when their size was reduced.

In order to make statistical comparison between any two conditions, a two-tailed Wilcoxon signed-ranks test was applied, comparing the error rates for each subject under these two conditions. (This test takes into consideration both the sign of the difference between each matched pair, and the rank ordering of the sizes of these differences; thus, the same result is obtained whether the test is based on absolute numbers of errors or on percentages of errors.)

It was found that the error rates in Condition $A$ were significantly lower than in Condition B, both for sequences where the standard and comparison tones were the same in pitch $[\mathrm{T}=0, \sigma \mathrm{T}=14.3, \mathrm{p}<.01]$ and also for sequences where they differed $[T=0$, $\sigma \mathrm{T}=11.25, \mathrm{p}<.01]$. The error rates in Condition $\mathrm{A}$ were also significantly lower than in Condition $\mathrm{C}$, both for sequences where the standard and comparison tones 
were the same in pitch $[\mathrm{T}=0, \sigma \mathrm{T}=8.44, \mathrm{p}<.01]$ and also for sequences where they differed $[\mathrm{T}=0$, $\sigma \mathrm{T}=12.75, \mathrm{p}<.01]$. Thus, in each case, repetition of the standard tone pitch resulted in a significant improvement in performance.

The error rate was also found to be significantly lower in Condition $\mathrm{C}$ than in Condition B for sequences where the standard and comparison tones were the same in pitch $[\mathrm{T}=0, \sigma \mathrm{T}=9.81, \mathrm{p}<.01]$ and this same difference was manifest as a nonsignificant trend in sequences where the standard and comparison tones differed in pitch $[\mathrm{T}=11.5, \sigma \mathrm{T}=8.44, \mathrm{p}>.05]$. Such an increase in error rate with an increase in the number of interpolated tones has been reported previously with this paradigm (Deutsch, 1970). The finding that substantially fewer errors occur when the standard tone pitch is repeated, but at the same time the total length of the interpolated sequence is increased, argues strongly that repetition produces true memory facilitation.

\section{EXPERIMENT II}

The purpose of Experiment II was to investigate the improvement in recognition performance produced by the repeated tone as a function of its position in the interpolated sequence. In the experiment of Deutsch (1972), a sequence of four tones was interpolated between the standard and comparison tones, and a tone of the same pitch as the standard tone was placed in either the second or third serial position of this sequence. The improvement in performance was greater at the second than at the third serial position, but this effect was small in size and did not reach statistical significance. So, in the present experiment, a longer tonal sequence was interpolated between the standard and comparison tones, and the effect of the repeated tone was studied at serial positions spaced farther apart. It was hoped in this way to obtain a significant and more substantial serial position effect.

\section{Methods}

Conditions. The experiment consisted of three conditions. In all conditions, the standard and comparison tones were separated by a sequence of six intervening tones. In Condition $A$, a tone of the same pitch as the standard tone was included in the second serial position of the intervening sequence. In Condition $B$, such a tone was included in the fifth serial position. In Condition $C$, no tone of the same pitch as the standard tone was included in the intervening sequence.

As in Experiment I, each condition consisted of 24 sequences; the conditions were identical to those in Experiment $I$ in all other respects.

Tonal Stimuli. These were the same as in Experiment I.

Subjects. Fifteen paid undergraduates at the University of California at San Diego served as subjects for the experiment. These were selected on the same criterion as for Experiment I.

Apparatus. This was the same as in Experiment I.

\section{Results}

As in Experiment $\mathrm{I}$, the percentage errors in each experimental condition were computed separately for each subject, averaged over two sessions, both for sequences where the standard and comparison tones were the same in pitch and for sequences where they differed. The percentage errors for each condition, averaged over all subjects, are displayed in Table 2 . It can be seen that a substantial decrease in errors occurred when a tone of the same pitch as the standard tone was placed in the second serial position of the intervening sequence, and a small decrease in errors occurred when this tone was placed in the fifth serial position.

Statistical comparisons were also made between the different experimental conditions, using two-tailed Wilcoxon tests, an in Experiment I. It was found that the error rate when the repeated tone was in the second serial position of the intervening sequence (Condition $\mathrm{A}$ ) differed significantly from baseline (Condition $\mathrm{C}$ ) both in sequences where the standard and comparison tones were the same in pitch $[\mathrm{T}=0, \sigma \mathrm{T}=12.75, \mathrm{p}<.01]$ and also in sequences where they differed $[\mathrm{T}=2.5$, $\sigma \mathrm{T}=12.75, \mathrm{p}<.01]$. However, the error rate when the repeated tone was in the fifth serial position (Condition B) dici not differ significantly from baseline (Condition C) either in sequences where the standard and comparison tones were the same in pitch $[T=16.5$, $\sigma \mathrm{T}=11.25, \mathrm{p}>.05]$ or in sequences where they differed $[\mathrm{T}=10, \sigma \mathrm{T}=9.81, \mathrm{p}>.05]$. Further, a significant difference in error rate was manifest depending on the serial position of the repeated tone (Conditions A vs. B) both in sequences where the standard and comparison tones were the same in pitch $[\mathrm{T}=0, o \mathrm{~T}=9.81, \mathrm{p}<.01]$ and in sequences where they differed $[\mathrm{T}=7.5$, $\sigma \mathrm{T}=12.75, \mathrm{p}<.02]$.

\section{DISCUSSION}

The results of the second experiment show that memory facilitation due to repetition is extremely sensitive to the position of the repeated tone in the intervening sequence. Although memory enhancement is substantial and highly significant when the repeated tone is placed in the second serial position of a sequence of

Table 2

Mean Percentages of Errors in Conditions of Experiment II

\begin{tabular}{|c|c|c|}
\hline \multirow[b]{3}{*}{ Condition } & \multicolumn{2}{|c|}{ Percent Errors } \\
\hline & \multicolumn{2}{|c|}{$\begin{array}{c}\text { Standard and } \\
\text { Comparison Tones }\end{array}$} \\
\hline & Same & Different \\
\hline $\begin{array}{l}\text { A. Standard tone pitch repeated } \\
\text { in second serial position of } \\
\text { intervening sequence. }\end{array}$ & 3.61 & 8.33 \\
\hline $\begin{array}{l}\text { B. Standard tone pitch repeated } \\
\text { in fifth serial position of inter- } \\
\text { vening sequence. }\end{array}$ & 9.72 & 15.00 \\
\hline $\begin{array}{l}\text { C. Standard tone pitch not } \\
\text { repeated in intervening } \\
\text { sequence. }\end{array}$ & 14.44 & 19.44 \\
\hline
\end{tabular}


six interpolated tones, it is small and insignificant when the repeated tone is placed in the fifth serial position. One might hypothesize from these findings that the interpolated tones produce a decrement in the trace of the standard tone which weakens its capacity to be strengthened by repetition.

The extreme sensitivity of repetition effects to disruption found here in memory for absolute pitch is in accordance with general experience, since only rare individuals are able to reproduce or recognize a tone in the musical scale, in spite of our daily exposure to a multitude of such tones. In contrast, most of us easily learn to recognize and recall tonal combinations such as melodies. However, the memories which are, in this case, enhanced by repetition are for abstracted relationships rather than for specific sets of tones (Attneave \& Olson, 1971; Deutsch, 1969). Posner (1969) and Craik \& Lockhart (1972) also argue from other examples that the abstraction of information leads to its retention in more enduring fashion.

The effect of amount of spacing between repetitions obtained here differs from effects reported in verbal memory. Waugh $(1963,1967)$ found no effect of amount of spacing between repetitions. Underwood (1969) obtained a similar result, with the exception of massed presentations. On the other hand, Melton (1967) and Glanzer (1969) found improved retention with increased spacing between repetitions. In contrast, the present study obtained poorer performance with increases spacing. Since all the above studies on verbal memory used the free recall procedure, the discrepancy with the present findings may simply reflect a difference in experimental paradigm. However, it is also quite possible that these differences reflect the functioning of different types of memory system.

\section{REFERENCES}

Attneave, F., \& Olson, R. K. Pitch as a medium: A new approach to psychophysical scaling. American Journal of Psychology, $1971,84,147-165$.
Besner, D., Keating, J. K. Cake, L. J. \& Maddigan, R. Repetition effects in iconic and verbal short term memory. Joumal of Experimental Psychology, 1974, 102, 901-903.

Craik, F. I. M., \& Lockhart, R. S. Levels of processing: A framework for memory research. Journal of Verbal Learning and Verbal Behavior, 1972, 11,671-684.

Crowder, R. G., \& Melton, A. W. The Ranschburg phenomenon: Failure of immediate recall correlated with repetition of elements within a stimulus. Psychonomic Science, 1965, 2, 295-296.

Deutsch, D. Music recognition. Psychological Review, 1969, 76, 300-307.

Deutsch, $D$. The deterioration of pitch information in memory. Unpublished doctoral dissertation, University of California at San Diego, 1970 .

Deutsch, D. Effect of repetition of standard and comparison tones on recognition memnrv for pitch. Journal of Experimental Psvchology, 1972, 93, 156-162.

Glanzer, M. Distance between related words in free recall: Trace of the STS. Journal of Verbal Learning and Verbal Behavior, $1969,8,105-111$.

Hintzman, D. L. Effects of repetition and exposure duration on memory. Journal of Experimental Psychology, 1970,83, 435-44.4.

Melton, A. W. Repetition and retrieval from memory. Science, $1967,158,532$.

Posner, M. I. Abstraction and the process of recognition. In G. H. Bower and J. T. Spence (Eds.) The psychology of learning and motivation: Advances in research and theory. Vol. $3, \mathrm{New}$ York: McGraw-Hill, 1969.

Sperling, $G$, The information available in brief visual presentations. Psychological Monographs, 1960, 74,(11 whole No. 498).

Standing, L. \& Da Polito, F. Limitations of the repetition effect revealed by partial report. Psychonomic Science, 1968, 13, 297-298.

Turvey, M. T, Repetition and the preperceptual information store, Journal of Experimental Psychology, 1967, 74, 289-293.

Underwood, B. J. Some correlates of item repetition in free-recall learning. Joumal of Verbal Learning and Verbal Behavior, $1969,8,83-94$

Waugh, N. C. The effect of intralist repetition of free recall. Joumal of Verbal Leaming and Verbal Behavior, 1962, 1. 95-99.

Waugh, N. C. Immediate memory as a function of repetition. Journal of Verbal Learning and Verbal Behavior, 1963,2, 107-112.

Waugh, N. C. Presentation time and free recall. Journal of Experimental Psychology, 1967, 73, 39-64.

Wolf, T. M., \& Jahnke, J. C. Effects of intraserial repetition on short-term recognition and recall. Journal of Experimental Psy chology, 1968, 77, 572-580.
(Received August 19, 1974; revision received November $1,1974$. 